\title{
S-duality and Topological Strings
}

\section{Citation}

Nekrasov, N, H Ooguri, and C Vafa. 2004. "S-Duality and Topological Strings." Journal of High Energy Physics 2004 (10): 009-009. https://doi.org/10.1088/1126-6708/2004/10/009.

\section{Permanent link}

http://nrs.harvard.edu/urn-3:HUL.InstRepos:41385019

\section{Terms of Use}

This article was downloaded from Harvard University's DASH repository, and is made available under the terms and conditions applicable to Other Posted Material, as set forth at http:// nrs.harvard.edu/urn-3:HUL.InstRepos:dash.current.terms-of-use\#LAA

\section{Share Your Story}

The Harvard community has made this article openly available.

Please share how this access benefits you. Submit a story.

Accessibility 
hep-th/0403167

CALT-68-2479

HUTP-04/A011

$\mathrm{IHES} / \mathrm{P} / 04 / 09$

ITEP-TH-62/03

\title{
S-duality and Topological Strings
}

\author{
Nikita Nekrasov, ${ }^{a \dagger}$ Hirosi Ooguri, ${ }^{b}$ and Cumrun Vafa $^{c}$ \\ ${ }^{a}$ Institut des Hautes Etudes Scientifiques \\ Bures-sur-Yvette, F-91440, France \\ ${ }^{b}$ California Institute of Technology, Pasadena, CA 91125, USA \\ c Jefferson Physical Laboratory, Harvard University \\ Cambridge, MA 02138, USA
}

\begin{abstract}
In this paper we show how S-duality of type IIB superstrings leads to an S-duality relating $\mathrm{A}$ and $\mathrm{B}$ model topological strings on the same Calabi-Yau as had been conjectured recently: D-instantons of the B-model correspond to A-model perturbative amplitudes and D-instantons of the A-model capture perturbative B-model amplitudes. Moreover this confirms the existence of new branes in the two models. As an application we explain the recent results concerning A-model topological strings on Calabi-Yau and its equivalence to the statistical mechanical model of melting crystal.
\end{abstract}

March 2004

$\dagger$ On leave of absence from: ITEP, Moscow, 117259, Russia 


\section{Introduction}

Topological strings come in many types, of which A and B models have been most thoroughly investigated. In the perturbative A model version Kähler geometry of the Calabi-Yau is probed while in the B model version the complex geometry of the Calabi-Yau is captured. On the other hand there is an intriguing role reversal when it comes to the Dbranes: The D-branes of A-model correspond to Lagrangian submanifolds, which naturally couple to the holomorphic 3-form, whereas the D-branes of the B-model correspond to holomorphic submanifolds, which naturally couple to Kähler structure.

In a recent paper [1] it was conjectured that this is not accidental and that there is an S-duality relating the A and B model topological strings on the same Calabi-Yau, where D-brane instanton amplitudes of the B-model are computed by worldsheet instantons of the A-model and non-perturbative A-model amplitudes correspond to the B-model perturbative amplitudes. This duality in particular predicts the existence of additional branes called "NS 5-brane" in the A-model and "NS 2-brane" in the B-model. This duality conjecture was motivated by explaining the equivalence of the B-model topological string on the twistorial Calabi-Yau $\mathbf{C P}^{3 \mid 4}$ [2] with the S-dual A-model topological string on the same Calabi-Yau. The two were conjectured to be related by a Montonen-Olive S-duality [1].

On the other hand a while back it was shown in [3] that the D1 and D(-1) brane instantons of the B-model are counted by the A-model worldsheet instantons. More recently a description of A-model on Calabi-Yau as the statistical mechanical model of melting crystal was discovered in [4. This was also interpreted as relating the A-model worldsheet instantons to D1 and D(-1)-branes which would make more sense in a B-model context [5,6]. Also, B model-like gauge-theoretic calculations were mapped to the A model worldsheet calculations with the topological gravity observables turned on in [7]. If there is an S-duality between A and B models on the same manifold these statements could be expected to be a consequence.

In this paper we explain how the S-duality of type IIB superstring in ten dimensions implies the S-duality between A and B model topological strings on the same manifold. Moreover we show how the results of [5, [6] may be viewed as an application of this S-duality. In particular the S-dual of D(-1) and D1 brane correspond to removing "atoms" or "edges"

1 To be precise, the S-duality of type IIB superstrings leads to the statement that D-brane instantons of B-model are captured by A-model worldsheet instantons. The reverse statement follows by mirror symmetry. 
from the Calabi-Yau crystal introduced in [4]. They are gravitational quantum foam in the A-model setup and can be interpreted as blowing up the A-model geometry along the branes.

It is natural to consider applications of these ideas to the twistorial Calabi-Yau in the absence of any extra branes, where we consider the pure gravitational theory: In particular the S-dual of D(-1) and D1 branes of the B-model on $\mathbf{C P}^{3 \mid 4}$ at B-model strong coupling are quantum foam in the twistor space $\mathbf{C P}^{3 \mid 4}$ in the weakly coupled A-model [8]2. Moreover in the B-model setting the D1 branes can be viewed as deforming the complex structure of twistor space [9] which according to the results of [10] can be mapped back to a quantum foam in $\mathbf{R}^{4}$. For example the D1 brane instanton wrapping a $\mathbf{P}^{1}$ cycle of the twistor space, gets mapped to blowing up a point in $\mathbf{R}^{4}[11,12]$. These are quite exciting as they would lead to a stringy description of quantum foam for $\mathcal{N}=4$ conformal supergravity! In fact some aspects of quantum foam for ordinary conformal gravity has been studied a long time ago [13].

The organization of this paper is as follows: In section 2 we review some basic facts about S-duality for type IIB superstring. In section 3 we show how this leads to an S-duality for topological strings mapping D-instantons of the B-model to worldsheet instantons of the A-model. In section 4 we consider an application of this idea and show how this leads to the picture proposed in [5,6] for computing A-model amplitudes in terms of Dinstantons. In section 5 we complete the discussion of S-duality by extending it to the mirror statement.

\section{S-duality for type IIB superstrings}

In this section we briefly recall certain aspects of S-duality for type IIB superstrings in ten dimensions which is relevant for us. We will be concentrating on the $\mathbf{Z}_{2} \subset S L(2, \mathbf{Z})$ subgroup of the S-duality group corresponding to strong/weak string coupling exchange. We will call the two dual theories by $B$ and $A$ (note that $A$ does not refer to type IIA superstring, but to a dual type IIB superstring- the reason for the choice of the letter $A$ becomes clear when we talk about topological strings in the next section).

2 This suggests that $\mathrm{D}(-1)$ brane instantons should also play a role in the twistorial Calabi-Yau proposed in [2]. 
Let us denote the superstring coupling constant by $g_{B}$. The strong/weak duality relates this to a dual type IIB coupling constant which we denote by $g_{A}$ :

$$
g_{A}=\frac{1}{g_{B}}
$$

As usual the coupling can be complexified, but we will deal with it in this way because all the relevant expressions are analytic. The metrics of the two theories are related by

$$
\frac{g_{\mu \nu}^{B}}{g_{B}}=g_{\mu \nu}^{A} .
$$

We will be interested in Calabi-Yau 3-fold backgrounds. It is natural to ask how the Kähler form $k$ and the holomorphic 3-form $\Omega$ of the Calabi-Yau transform under S-duality. By $\Omega$ we mean the normalized holomorphic three form such that $\Omega \wedge \bar{\Omega}_{0}$ gives the volume form. Here we vary the holomorphic form $\Omega$ while keeping $\bar{\Omega}_{0}$ fixed and so all the volume dependence in captured by the holomorphic part $\Omega$ (i.e. we can view $\Omega$ as mirror to the Kähler volume $k \wedge k \wedge k$ ). From (2.2) it follows that

$$
\begin{aligned}
& \frac{k_{B}}{g_{B}}=k_{A} \\
& \frac{\Omega_{B}}{g_{B}^{3}}=\Omega_{A}
\end{aligned}
$$

Under S-duality we have the following exchange of the branes

$$
\begin{gathered}
D 1 \leftrightarrow F 1 \\
D 5 \leftrightarrow N S 5
\end{gathered}
$$

where $F 1$ denotes the fundamental string. It will be convenient to discuss what holomorphic configurations of $D 1$ branes and $F 1$ branes couple to. For $D 1$ brane this is given by

$$
\int_{D 1} \frac{k_{B}}{g_{B}}+i B_{R}
$$

where the first term comes from the volume of $D 1$ brane and the second term denotes the fact that $D 1$ brane is charged under the RR 2-form field $B_{R}$. Similarly holomorphic $F 1$ couples to

$$
\int_{F 1} k_{A}+i B_{N S}
$$


where $B_{N S}$ denotes the NS-NS 2-form field. It is natural to define the fields

$$
\begin{gathered}
\hat{k}_{B}=k_{B}+i g_{B} B_{R} \\
\hat{k}_{A}=k_{A}+i B_{N S}
\end{gathered}
$$

in terms of which the holomorphic $D 1$ brane couples to $\hat{k}_{B} / g_{B}$ and the holomorphic $F 1$ branes couple to $\hat{k}_{A}$. Under S-duality we have the map

$$
\frac{\hat{k}_{B}}{g_{B}}=\hat{k}_{A}
$$

reflecting the fact the $D 1$ and $F 1$ exchange under S-duality. This is what replaces (2.3) when the B-fields are turned on. For simplicity of notation for the rest of the paper we drop the hats and denote the Kähler forms, including the $B$ fields by $k_{B}$ and $k_{A}$.

\section{Type IIB superstrings and A and B model topological strings}

Consider type IIB superstrings compactified on a Calabi-Yau threefold $M$. Consider the topological strings on $M$. There are two versions, $A$ and $B$, of topological strings on $M$ and they are known to compute 'F-terms' for type IIB superstrings: B-model topological strings compute F-terms for vector multiplets [14, 15], and A-model topological strings computes F-terms for hypermultiplets of type IIB superstrings [15]. For each worldsheet genus $h$, each topological string computes correction for a different, but unique F-term. Moreover the topological string amplitude at given genus corresponds to the F-term correction in the superstring coming from the same genus amplitude. Thus in this way we can identify the topological string coupling constant with the superstring coupling constant, bearing in mind that each term of the topological string computes different amplitudes for the superstring. In some cases these different terms can be identified, as in the context of black holes [16, 17].

Consider topological B-model. We ask if there are any non-perturbative corrections. Let us consider the ones coming from D1 brane instantons. These are corrections to the hypermultiplet moduli. As discussed in the previous section these couple to $k_{B} / g_{B}$. However, under S-duality D1 brane and F1 brane are exchanged, and the corresponding instantons get mapped to holomorphic worldsheet instantons. These are precisely the objects of relevance for the A-model topological string! Thus these D-brane instanton corrections are mapped to A-model perturbative worldsheet correction of the dual theory. 
We are thus led to view the non-perturbative completion of B-model as including the perturbative modes of the dual A-model. Moreover the D1 brane instantons of the Bmodel are captured by the dual worldsheet A-model instantons on the same manifold. This was conjectured in [1] and we now see it can be inferred from superstring S-duality for type IIB superstrings. In particular we have

$$
\begin{gathered}
g_{A}=1 / g_{B} \\
k_{A}=k_{B} / g_{B}
\end{gathered}
$$

What is the interpretation of the various D-branes in the dual A-model? The interpretation of D5 brane dual is an NS 5-brane. This provides evidence for the existence of a new brane in A-model, which was conjectured in [1]. It is also natural to expect that a holomorphic Chern-Simons theory lives on this NS 5-brane, as would follow from type IIB string duality.

The story for D1 branes and $\mathrm{D}(-1)$ brane instantons are expected to be more tricky as we know from superstrings. Consider in particular a contribution in the B-model involving $N \mathrm{D}(-1)$ brane instantons and a D1 brane wrapped over a 2-cycle class $(c)$. These should contribute to the amplitude by a factor of

$$
\exp \left[-N / g_{B}-k_{B}(C) / g_{B}\right]=\exp \left[-N g_{A}-k_{A}(C)\right]
$$

This factor for the A-model is not the usual one and in particular the $g_{A}$ appears in a very different way from the conventional form. However in fact this form of the contribution anticipates a periodicity in $g_{A}$ by shifting by $g_{A} \rightarrow g_{A}+2 \pi i$ which was predicted in [3] based on considerations of embedding topological string in superstrings and viewing it as counting wrapped D2 brane degeneracies. Thus it means that $\mathrm{D}(-1)$ brane and D1 branes repackage the A-model amplitudes in a different way.

In fact the story is much better: This form of the A-model expansion is deeply connected to the recent discovery of the description of A-model topological strings on CalabiYau as a melting crystal [4] reformulated as a $U(1)$ gauge theory [5,6]. Moreover in that context the $\mathrm{D}(-1)$ brane and D1 brane instantons did end up having a geometric meaning in the dual A-model context: They correspond to quantum gravitational foam for the A-model, where the space was blown up along the location of $\mathrm{D}(-1)$ brane points and D1 brane curves of the size $g_{A}$. Thus this gives a more precise meaning as to the role of $\mathrm{D}(-1)$ and D1 brane instantons viewed from the dual A-model. 
So far we have only shown that S-duality predicts a duality between D1 and D(-1)brane instantons of the B-model and perturbative amplitudes of the A-model, and have made some qualitative checks. Can we use this duality to gain further insight into the A-model amplitudes? In particular can we use the D-instanton sum of the B-model as a new way to compute the A-model amplitudes?

In fact we will argue in the next section that this is possible and that it has already been done [5,6]!

\section{Holomorphic Chern-Simons and A-model}

In the previous section we have argued that the A-model amplitudes should be computable by summing up the $\mathrm{D}(-1)$ and $\mathrm{D} 1$ brane instantons. The natural question is how to compute these instanton contributions directly.

There is one natural way this can be done. We can consider a single D5 brane wrapped over the Calabi-Yau. In this context the sum over the $\mathrm{D}(-1)$ and $\mathrm{D} 1$ brane instantons is the same as the sum over the various sectors of the $U(1)$ bundle. On the D5 brane, in the topological B-model context, lives a $U(1)$ holomorphic Chern-Simons theory [18]. Moreover the $N D(-1)$ branes and $D 1$ brane wrapped over a cycle $[C]$ get mapped to a gauge theory configuration for holomorphic Chern-Simons having

$$
\begin{gathered}
c h_{3}=N \\
c h_{2}=[C] .
\end{gathered}
$$

In particular the " $U(1)$ " theory is a stringy $U(1)$ theory which supports such non-trivial configurations. Of course holomorphic Chern-Simons will also have perturbative contributions. These will not be relevant for the above instanton terms, if we wish to compute A-model closed string amplitudes. So roughly speaking we wish to compute

$$
Z_{h C S} / Z_{h C S}^{\text {pert. }}
$$

and we are organizing the instanton sum, using (3.1) as

$$
\exp \left[-g_{A} \int c h_{3}-\int k_{A} \wedge c h_{2}\right]
$$

We thus expect to have a $U(1)$ gauge theory with the above weight which is morally the non-perturbative contributions of the holomorphic Chern-Simons. 
There are two proposals for what this gauge theory may be [5,6]. The formulation in [5] involves a twisted version of maximally supersymmetric Yang-Mills on the Calabi-Yau. The one in [6] is morally equivalent to holomorphic Chern-Simons theory on the CalabiYau (in the sense that it "counts" holomorphic bundles defined mathematically in [19]). It is not known if the two are equivalent. Both of them localize to holomorphic bundles (or more precisely ideal sheaves) on the Calabi-Yau.

Let us discuss the one in [5] from the perspective of the present paper: If we think about the D5 brane in the superstring, it supports the partially twisted maximally supersymmetric Yang-Mills theory. Its instanton equations (for $N$ D5 branes) are given by:

$$
\begin{aligned}
& F_{A}^{2,0}=\bar{\partial}_{A}^{\dagger} \varphi \\
& F_{A}^{0,2}=\partial_{A}^{\dagger} \bar{\varphi} \\
& F_{A}^{1,1} \wedge k^{2}=[\varphi, \bar{\varphi}]+\lambda \cdot k^{3}
\end{aligned}
$$

where $\varphi$ is the adjoint-valued $(3,0)$-form on the worldvolume of the brane, which is the twisted complex Higgs field of the six dimensional gauge theory. The equations (4.1) depend on the choice of complex structure, and Kähler structure. However, as it often happens with the twisted gauge theories, small variations of the Kähler structure should not affect gauge theory correlation functions.

Moreover, on Calabi-Yau manifolds, on the solutions of (4.1) the (3,0)-form vanishes, $\varphi=0$. Then the equations (4.1) can be reformulated as $F_{A}^{0,2}=0$ and its conjugate, while the last equation together with ordinary $U(N)$ gauge symmetry combine to the complexified gauge symmetry, $G L(N, \mathbf{C})$. But then we are discussing precisely the equations of motion of the holomorphic Chern-Simons theory on the same manifold, modulo complex gauge transformations, which is the gauge symmetry of hCS!

We should be more careful, though. In the physical D5 brane theory there are four scalars, of which two are twisted into $\varphi, \bar{\varphi}$, while the other two remain intact, $\Phi, \bar{\Phi}$. The contribution of the instanton solution (4.1) to the gauge theory path integral is given by the ratio of determinants:

$$
\frac{\operatorname{Det}_{\Omega^{0,0}} A d(\Phi) \operatorname{Det}_{\Omega^{0,2}} A d(\Phi)}{\operatorname{Det}_{\Omega^{0,1}} A d(\Phi) \operatorname{Det}_{\Omega^{0,3}} A d(\Phi)}
$$

where the numerator comes from Faddeev-Popov ghosts and the fermions which couple to the equations (4.1), while the denominator comes from the fluctuations of the gauge fields $A$ and Higgs $\varphi$. Now, the remarkable fact is that on Calabi-Yau manifolds the ratio of the determinants (4.2) is actually $\Phi$-independent, apart from inessential universal 
perturbative piece [5,6]. We present some details of this computation in the Appendix.

This makes it possible to identify the instanton contributions with those of hCS theory, as we had anticipated from the S-duality of topological strings.

\section{Completing the S-duality of A and B models}

So far we have argued that the non-perturbative amplitudes of the B-model are captured by perturbative amplitudes of the A-model. To complete the story we need to argue, as has been conjectured in [1], that the non-perturbative amplitudes of the A-model are computed by the perturbative amplitudes of the B-model. This is clearly plausible, because the instantons of the A-model are D2 branes wrapped over Lagrangian 3-cycles of CY. Moreover one expects that the perturbative B-model "counts" D3 branes wrapped over Lagrangian 3-cycle. We will now present further arguments to support this. In fact this follows from mirror symmetry. If the non-perturbative amplitude of B-model is computed by perturbative amplitudes of the A-model, then the mirror statement is that the non-perturbative amplitude of the A-model is computed by perturbative amplitudes of the B-model. Thus to argue this statement it is natural to consider type IIA superstrings on a Calabi-Yau.

So consider type IIA superstring compactified on the Calabi-Yau $M$. To this end it is natural to promote the A-model threeform $\Omega_{A}$ to

$$
\hat{\Omega}_{A}=\Omega_{A}+i g_{A} C_{R}
$$

where $C_{R}$ is an RR 3-form field for type IIA superstrings. The analog of S-dual of $C_{R}$ is a $N S$ field $C_{N S}$ whose flux corresponds to non-integrability of the complex structure. This was anticipated by mirror symmetry [20] and has been verified in a number of examples [21,22,23]. The dual three form is defined by

$$
\hat{\Omega}_{B}=\Omega_{B}+i g_{B}^{2} C_{N S}
$$

This is forced by the condition that $C_{R} \leftrightarrow C_{N S}$ under the topological S-duality, and the fact that (2.4) predicts

$$
\frac{\hat{\Omega}_{B}}{g_{B}^{2}} \leftrightarrow \frac{\hat{\Omega}_{A}}{g_{A}} .
$$

Note that the fact that in the A-model the worldsheet instantons can end on Lagrangian D-branes, gets mapped by the S-duality to the statement that the D1 brane instantons 
can end on Lagrangian NS branes, which was called "NS 2-branes" in [1]. Their geometric meaning is that they correspond to a source for lack of integrability of the complex structure of the Calabi-Yau in the B-model.

Let us drop the hats from $\Omega$ 's keeping in mind that we can add these fields. As further evidence for this duality, let us recall the term considered in [1]: It was argued there that there is a term in the topological B-model given by

$$
\int \frac{\Omega_{B} \wedge d k_{B}}{g_{B}^{2}}
$$

note that this includes the term $\frac{i}{g_{B}} \int \Omega_{B} \wedge d B_{R}$. Another way to explain the existence of this term is to note that this is a superpotential term generated by RR flux $H_{R}=d B_{R}$ [24,25]. The S-dual of the above term, using the above transformations is given by

$$
\int \frac{\Omega_{A} \wedge d k_{A}}{g_{A}^{2}}
$$

which is also generated in the A-model as explained in [1].

That perturbative B-model can be reformulated as a sum over Lagrangian D-branes is amusing and it would be interesting to see if this leads to another computational scheme for the B-model.

\section{Acknowledgments.}

We would like to thank M. Aganagic, S. Gukov, A. Kapustin, S. Katz, L. Motl, A. Neitzke, A. Okounkov, A. Strominger and C. Taubes for valuable discussions. In addition NN would like to thank A. Losev 3 for interesting discussions.

The research of NN is partly supported by РФФИ grant 03-02-17554 and by the grant HШ-1999.2003.2 for scientific schools. The research of HO is supported in part by DOE grant DE-FG03-92-ER40701. The research of CV is supported in part by NSF grants PHY-0244821 and DMS-0244464.

3 In fact, A. Losev has been advocating the idea of S-dualities and even M-theories in the context of topological strings for a while [26]. 


\section{Appendix A. Instanton contribution}

We consider the instanton contribution to the partition function of the maximally supersymmetric $U(N)$ gauge theory on $\mathbf{R}^{6}$. In order to regularize the possible infrared divergencies we shall work equivariantly with respect to the rotations of $\mathbf{R}^{6}$. More precisely, in order to preserve some fermionic symmetry one should combine the rotations of $\mathbf{R}^{6}$ with some R-symmetry rotations. The simplest possibility is to compensate rotation generated by the $S O(6)$ matrix

$$
\Omega=\left(\begin{array}{cccccc}
0 & \epsilon_{1} & & & & \\
-\epsilon_{1} & 0 & & & & \\
& & 0 & \epsilon_{2} & & \\
& & -\epsilon_{2} & 0 & & \\
& & & & 0 & \epsilon_{3} \\
& & & & -\epsilon_{3} & 0
\end{array}\right)
$$

by twisting one of the complex scalars $\varphi$ by $e^{-i\left(\epsilon_{1}+\epsilon_{2}+\epsilon_{3}\right)}$. The remaining scalar, $\Phi$, is the Higgs field and could potentially enter the instanton contributions, thus spoiling the conjectured hCS/SYM duality.

In fact, the contribution of a given instanton is then given by:

$$
\exp \int_{0}^{\infty} \frac{d t}{t} \frac{\operatorname{Tr} e^{t \Phi} \operatorname{Tr} e^{-t \Phi}}{\left(1-e^{t \epsilon_{1}}\right)\left(1-e^{t \epsilon_{2}}\right)\left(1-e^{t \epsilon_{3}}\right)}
$$

and

$$
\operatorname{Tr} e^{t \Phi}=\sum_{l=1}^{N} e^{t a_{l}}\left[1-\left(1-e^{t \epsilon_{1}}\right)\left(1-e^{t \epsilon_{2}}\right)\left(1-e^{t \epsilon_{3}}\right) \sum_{(i, j, k) \in \pi_{l}} e^{t\left(\epsilon_{1}(i-1)+\epsilon_{2}(j-1)+\epsilon_{3}(k-1)\right)}\right],
$$

where $\epsilon_{1}, \epsilon_{2}, \epsilon_{3}$ are the equivariant parameters of the $S O(6)$ rotation. They can also be mapped to the components of the field strength of the graviphoton field. The $U(N)$ instantons are labeled by the $N$-tuples of three dimensional partitions $\pi_{l}, l=1, \ldots, N$ and the classical value of the Higgs field $\Phi$ is given by the diagnal matrix with entries $\left(a_{1}, \ldots, a_{N}\right)$. The extra terms in (A.3) are the quantum corrections, which lead to the modified expressions for the $\left\langle\operatorname{Tr} \Phi^{k}\right\rangle$ expectation values.

Now the crucial point, heavily exploited in [6] [5] is that for the Calabi-Yau choice of the rotation parameters, i.e. for $\epsilon_{1}+\epsilon_{2}+\epsilon_{3}=0$ the determinants (A.2) almost cancel, 
leaving only the overall sign $(-)^{\sum_{l}\left|\pi_{l}\right|}$ and the universal perturbative factor independent of $\pi_{l}$ :

$$
\begin{aligned}
& Z^{\text {pert }}=\exp \sum_{l, m} \gamma\left(\frac{a_{l}-a_{m}}{\Lambda}\right) \\
& \gamma(x)=\left.\frac{d}{d s}\right|_{s=0}\left(\frac{1}{\Gamma(s)} \int_{0}^{\infty} \frac{d t}{t} t^{s} \frac{e^{-t x}}{\left(1-e^{t \epsilon_{1}}\right)\left(1-e^{t \epsilon_{2}}\right)\left(1-e^{t \epsilon_{3}}\right)}\right) .
\end{aligned}
$$




\section{References}

[1] A. Neitzke and C. Vafa, "N $\mathcal{N}=2$ strings and the twistorial Calabi-Yau," hepth/0402128.

[2] E. Witten, "Perturbative gauge theory as a string theory in twistor space," hepth/0312171.

[3] R. Gopakumar and C. Vafa, "M-theory and topological strings. I," hep-th/9809187; "M-theory and topological strings. II," hep-th/9812127.

[4] A. Okounkov, N. Reshetikhin, and C. Vafa, "Quantum Calabi-Yau and classical crystals", hep-th/0309208.

[5] A. Iqbal, N. Nekrasov, A. Okounkov, and C. Vafa, "Quantum foam and topological strings," hep-th/0312022.

[6] D. Maulik, N. Nekrasov, A. Okounkov, and R. Pandharipande, "Gromov-Witten theory and Donaldson-Thomas theory," math.AG/0312059.

[7] A. Losev, A. Marshakov, and N. Nekrasov, "Small instantons, little strings and free fermions," hep-th/0302191, in: From fields to strings: circumnavigating theoretical physics, Ian Kogan Memorial Volume, M. Shifman, A. Vainshtein, and J. Wheater eds., World Scientific, Singapore.

[8] C. Vafa, work in progress.

[9] M. Aganagic, R. Dijkgraaf, A. Klemm, M. Marino, and C. Vafa, "Topological strings and integrable hierarchies," hep-th/0312085.

[10] M.F. Atiyah, N.J. Hitchin, and I.M. Singer, "Self-duality in four dimensional Riemannian geometry," Proc. Roy. Soc. London Ser. A 362, 425 (1978).

[11] C.H. Taubes, "The Existence of anti-self-dual conformal structures," J. Diff. Geom. 36, 163 (1992).

[12] S.K. Donaldson and R. Friedman, "Connected sums of self dual manifolds and deformations of singular spaces," Nonlinearity 2, 197 (1989).

[13] A. Strominger, Phys. Rev. Lett. 52, 1733 (1984).

[14] M. Bershadsky, S. Cecotti, H. Ooguri, and C. Vafa, "Kodaira-Spencer theory of gravity and exact results for quantum string amplitudes," Commun. Math. Phys. 165 (1994) 311-428, hep-th/9309140.

[15] I. Antoniadis, E. Gava, K. S. Narain, and T. R. Taylor, "topological amplitudes in string theory," Nucl. Phys. B413 (1994) 162-184, hep-th/9307158.

[16] G. Lopes Cardoso, B. de Wit, and T. Mohaupt, "Deviations from the area law for supersymmetric black holes," Fortsch. Phys. 48, 49 (2000), hep-th/9904005.

[17] H. Ooguri, A. Strominger, and C. Vafa, work to appear.

[18] E. Witten, "Chern-Simons gauge theory as a string theory," Prog. Math. 133, 637 (1995), hep-th/9207094. 
[19] S. Donaldson and R. Thomas, "Gauge theory in higher dimensions," in The geometric universe: science, geometry and the work of Roger Penrose, S. Huggett et. al. eds., Oxford Univ. Press, 1998.

[20] C. Vafa, "Superstrings and topological strings at large N," J. Math. Phys. 42, 2798 (2001), hep-th/0008142.

[21] S. Gurrieri, J. Louis, A. Micu, and D. Waldram, "Mirror symmetry in generalized Calabi-Yau compactifications," Nucl. Phys. B 654, 61 (2003), hep-th/0211102.

[22] S. Kachru, M. B. Schulz, P. K. Tripathy, and S. P. Trivedi, "New supersymmetric string compactifications," JHEP 0303, 061 (2003), hep-th/0211182.

[23] T.W. Grimm and J. Louis, "The effective action of $\mathcal{N}=1$ Calabi-Yau orientifolds," hep-th/0403067.

[24] S. Gukov, C. Vafa, and E. Witten, "CFT's from Calabi-Yau four-folds," Nucl. Phys. B 584, 69 (2000) [Erratum-ibid. B 608, 477 (2001)], hep-th/9906070.

[25] T. R. Taylor and C. Vafa, "RR flux on Calabi-Yau and partial supersymmetry breaking," Phys. Lett. B 474, 130 (2000), hep-th/9912152.

[26] A. S. Losev, "Perspectives of string theory," talk at the "String theory at Greater Paris" seminar, 2001. 